\title{
Development of A New Algorithm For Optimal Solution Of Transportation Problems
}

\author{
I.A. Rajper ${ }^{++}$. S. F. Shah* . A. S. Qureshi \\ Department of Basic Science and Related Studies, Mehran University of Engineering and Technology \\ ${ }^{++}$Correspondence with Author: Aliimranrajper@gmail.com
}

\begin{abstract}
This paper leads to an Algorithm/technique to solve optimal solution occurring in Transportation problems. In Transportation Problems by presenting a new algorithm to choose the Absolute differences of boundary cost cells. This New Algorithm lesser time than the existing Transportation method to get the optimal solution using initial basic feasible solution. Proposed technique/algorithm is better choice to get optimal solution without finding initial basic feasible solution and hence the proposed algorithm is useful to get optimal solution Transportation problems.
\end{abstract}

Key words:- Transportation Problems, Absolute differences of boundary cost cells, Modified Distribution (M.O.D.I) Method, Initial Basic feasible solution, Optimum solution.

DOI: $10.7176 / \mathrm{MTM} / 9-1-07$

\section{Introduction:-}

In Mathematics Operation Research (OR) is an application of scientific methods which deals with problems, formulations, solutions and finally taken better decision making.

Linear programming is a powerful tool of operational research methodology to solve allocation problem. It is the method used in decision making, especially in business management and government administration for obtaining the "Minimum or Maximum" value of an objective function according to given linear constraints. Linear programming deals with many problems such as Allocation Problems, Diet Problems, Transportation Problems, Agriculture Problems, Research \& Development, Marketing Engineering, Management Sciences, Medical Sciences, Network Problems, Logistics, Statistics, and many others [1]. "The basic Transportation problem" originally developed by (Hitch cock 1941).

The main purpose of transportation problem is to minimize the transportation cost. In other words, Transport different quantities of a single uniform product to different destinations in such a way that total transportation cost is minimum. There are two types of transportation problems (TP). 
Type-1: "Balanced transportation problem" in which the total supply equals to the total demand.

Type-2: "Unbalanced transportation problem" When total supply and total demand is not equal.

There are two phases to get solution transportation problems (TP).

Phase-1: To achieve the "Initial basic feasible solution (IBFS) Transportation Problems".

Phase-2: To obtain the optimal solution of Transportation Problems [2].

Ashraful babu [3] gave a new method to finding the "Initial basic feasible solution" of a Transportation Problem (TP). In which author gave a new method called "Lowest Allocation Method (LAM)".This proposed method is easier than other methods to compute feasible solution. M.A Metlo [4] introduced a new method named as "Modified North West Corner" based on "North West Corner" to compute feasible solution. The result of this work was reducing the size of iterations. According to the time point of view purposed technique was more powerful than North West Corner. Tuncay [5] According to this Author, author was introduced a new approximation method called "Tuncay can's approximation method". The main objective of proposed method was only used for finding "Initial basic feasible solution (IBFS) of balanced Transportation problem". Author was said proposed method takes usually less number of iterations and gave optimal solution other than approximation method of Transportation Problems. G.Kalpana [6] percent discussion main problem behind the grid computing environment was arrangement a job and mapping the user jobs to the resources. New method was a better form of "Divisible Load Theory" method. The proposed work is comparatively $39.83 \%$ better than the other existing method. Ashraful Babu [7] describe an algorithm proposed work gave very close to optimal solution but many times gave equal to optimal solution. New proposed method was not sure for all times "I.C.M" gave smallest feasible solution but a good number of the times it gave better result. Ab. Sattar [8] proposed an algorithm for "Initial basic feasible solution" of transportation model. His "Modified Vogel's Approximation Method" (MVAM) gave same result as (VAM), but batter then (NWCM) and (LCM). Proposed method is also easily applied to balanced and unbalanced Transportation models. D. Almaatani [9] Aim of proposed work was to find optimal solutions to linear transportation problems. Agarana M. [10] A case study of "Enhancing the Movement of People and Goods in A Potential World Class University using Transportation Model". The optimal solution shows that the group of people and goods in Covenant University to use less of time spent to move from one point to another. Sharif Uddin [11] introduce new algorithm 
to obtain the batter "Initial basic feasible solution" of Transportation Problem (TP). The aim of this study is to compare the result of proposed method with other existing methods but (ILCM) gave batter (IBFS) and closest to optimal solution.

\section{Problem Statement \& Methodology:}

The general Mathematical formulation of transportation problem is given as:

$$
\begin{aligned}
\text { Minimized } Z= & \sum_{i=1}^{p} \sum_{j=1}^{q} C_{i j} X_{i j} \\
\text { Subject to: } & \sum_{i=1}^{p} X_{i j}=S_{i} \\
& \sum_{j=1}^{q} X_{i j}=d_{j}
\end{aligned}
$$

(Total Tranasportation Cost )

(Supply from source i )

(demand from destination $\mathbf{j}$ )

$$
\text { Where } \quad x_{i j} \geq 0 \quad \text { for } \forall i \text { and } j
$$

\section{Algorithm of (M.O.D.I) Method:-}

1) Find a Initial Basic Feasible Solution using (N.W.C.M),(L.C.M),(V.A.M) etc.

2) Find $U$ 's \& V's Values Using Formula $U_{i}+V_{j}=C_{i j}$ for all allocated cells.

3) Now find $P_{i j}=U_{i}+V_{j}-C_{i j}$ for all non allocated cells. If $P_{i j}=U_{i}+V_{j}-C_{i j} \leq 0$ then stop required solution is optimal.

4) Using New Basic Feasible Solution to repeat step (2) \& (3) until $P_{i j}=U_{i}+V_{j}-C_{i j} \leq 0$ is true.

\section{Propose New Algorithm:-}

1) Take the Absolute differences of boundary cost cells of cost matrix like as:

$$
\begin{array}{lll}
> & \left|\mathbf{C}_{11}-\mathbf{C}_{1 q}\right|=\mathbf{A}_{1} \\
> & \left|\mathbf{C}_{11}-\mathbf{C}_{1 p}\right|=\mathbf{A}_{2} \\
> & \left|\mathbf{C}_{1 p}-\mathbf{C}_{\text {pq }}\right|=\mathbf{A}_{3} \\
> & \left|\mathbf{C}_{1 q}-\mathbf{C}_{\text {pq }}\right|=\mathbf{A}_{4}
\end{array}
$$

2) Let $A_{1}$ Maximum Absolute difference.

3) Let $\mathrm{C}_{11}$ is the smallest unit of cost cell in Row / Column.

4) Allocate the Minimum amount according to Supply/ Demand.

5) Repeat step (1) To (4) when Supply \& Demand become zero. 


\section{Numerical Examples:-}

In this paper, consider different - size of Transportation Problems, selected from above literature. We also use these examples to perform a comparative study of proposed algorithm with M.O.D.I , N.W.C.M and L.C.M. We Solve example -1 step-by-step continuous

\begin{tabular}{|c|c|c|c|c|c|c|}
\hline \multirow{6}{*}{ Exaple\#1 } & SOURSE & \multicolumn{4}{|c|}{ DESTINATION } & \multirow[t]{2}{*}{ SUPPLY } \\
\hline & & $\mathbf{D}_{1}$ & $\mathbf{D}_{2}$ & $\mathbf{D}_{3}$ & $\mathbf{D}_{4}$ & \\
\hline & $\mathbf{S}_{1}$ & 3 & 1 & 7 & 4 & 250 \\
\hline & $\mathbf{S}_{2}$ & 2 & 6 & 5 & 9 & 350 \\
\hline & $\mathbf{S}_{3}$ & 8 & 3 & 3 & 2 & 400 \\
\hline & DEMAND & 200 & 300 & 350 & 150 & $=$ \\
\hline \multirow{6}{*}{ Exaple\#2 } & SOURSE & \multicolumn{4}{|c|}{ DESTINATION } & SUPPLY \\
\hline & & $\mathbf{D}_{1}$ & $\mathbf{D}_{2}$ & $\mathbf{D}_{3}$ & $\mathbf{D}_{4}$ & \\
\hline & $\mathbf{S}_{1}$ & 3 & 1 & 7 & 4 & 300 \\
\hline & $\mathbf{S}_{2}$ & 2 & 6 & 5 & 9 & 400 \\
\hline & $\mathbf{S}_{3}$ & 8 & 3 & 3 & 2 & 500 \\
\hline & DEMAND & 250 & 350 & 400 & 200 & $=$ \\
\hline
\end{tabular}

\section{Example\#1:-}

Consider a Mathematical Model of a Transportation Problem in bellow Table.

\begin{tabular}{|c|c|c|c|c|c|}
\hline \multirow[t]{2}{*}{ SOURSE } & \multicolumn{4}{|c|}{ DESTINATION } & \multirow[t]{2}{*}{ SUPPLY } \\
\hline & $\mathbf{D}_{1}$ & $\mathbf{D}_{2}$ & $\mathbf{D}_{3}$ & $\mathbf{D}_{4}$ & \\
\hline $\mathbf{S}_{1}$ & 3 & 1 & 7 & 4 & 250 \\
\hline $\mathbf{S}_{2}$ & 2 & 6 & 5 & 9 & 350 \\
\hline $\mathbf{S}_{3}$ & 8 & 3 & 3 & 2 & 400 \\
\hline DEMAND & 200 & 300 & 350 & 150 & $=$ \\
\hline
\end{tabular}

Solution of Example- 1 using MODI Method:-

Step \# 1:- find initial basic feasible solution using (NWCM):-

\begin{tabular}{|c|c|c|c|c|}
\hline $\begin{array}{l}3 \\
200\end{array}$ & 1 & 7 & 4 & $\begin{array}{l}250 \\
50\end{array}$ \\
\hline 2 & 6 & 5 & 9 & 350 \\
\hline 8 & 3 & 3 & 2 & 400 \\
\hline $\begin{array}{l}20 \theta \\
\theta\end{array}$ & 300 & 350 & 150 & \\
\hline
\end{tabular}

\begin{tabular}{|c|c|c|c|}
\hline 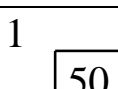 & 7 & 4 & $\begin{array}{l}50 \\
\theta\end{array}$ \\
\hline 6 & 5 & 9 & 350 \\
\hline 3 & 3 & 2 & 400 \\
\hline $\begin{array}{l}300 \\
250\end{array}$ & 350 & 150 & \\
\hline
\end{tabular}

\begin{tabular}{|c|c|c|c|}
\hline$\sqrt[6]{250}$ & 5 & 9 & $\begin{array}{l}350 \\
100\end{array}$ \\
\hline 3 & 3 & 2 & 400 \\
\hline $\begin{array}{l}250 \\
0\end{array}$ & 350 & 150 & \\
\hline
\end{tabular}

\begin{tabular}{|c|c|c|c|c|c|}
\hline$\sqrt[5]{100}$ & 9 & $\begin{array}{l}10 \theta \\
0\end{array}$ & 3 & 2 & $\begin{array}{l}400 \\
150\end{array}$ \\
\hline 3 & 2 & 400 & 250 & 150 & \\
\hline 350 & 150 & & 0 & & \\
\hline
\end{tabular}

\begin{tabular}{|l|l|}
\hline 2 & 150 \\
\hline 150 & 0 \\
\hline 150 & \\
0 & \\
\hline
\end{tabular}


Step \# 2:- Find U's \& V's Values Using Formula $U_{i}+V_{j}=C_{i j}$ for all allocated cells.

\begin{tabular}{|c|c|c|c|c|c|}
\hline & $V_{1}=3$ & $\mathrm{~V}_{2}=1$ & $\mathrm{~V}_{3}=0$ & $V_{4}=-1$ & $S$ \\
\hline $\mathrm{U}_{1}=0$ & $\begin{array}{r}3(-) \\
200\end{array}$ & 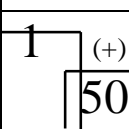 & 7 & 4 & 250 \\
\hline $\mathrm{U}_{2}=5$ & $22_{(+)}$ & 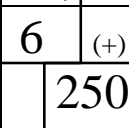 & $\sqrt[5]{100}$ & 9 & 350 \\
\hline $\mathrm{U}_{3}=3$ & 8 & 3 & ${ }^{3}$ & $\stackrel{2}{150}$ & 400 \\
\hline $\mathrm{D}$ & 200 & 300 & 350 & 150 & \\
\hline
\end{tabular}

Set $U_{1}=0 U_{i}+V_{j}=C_{i j}$ for all allocated cells. Step \#3: Now find $\mathbf{P}_{\mathbf{i j}}=\mathbf{U}_{\mathbf{i}}+\mathbf{V}_{\mathbf{j}}-\mathbf{C}_{\mathbf{i j}}$ for all non

$$
\begin{aligned}
& \mathrm{U}_{1}+\mathrm{V}_{1}=\mathrm{C}_{11} \Rightarrow 0+\mathrm{V}_{1}=3 \quad \mathrm{~V}_{1}=3 \\
& \mathrm{U}_{1}+\mathrm{V}_{2}=\mathrm{C}_{12}=>0+\mathrm{V}_{2}=1 \quad \mathrm{~V}_{2}=1 \\
& \mathrm{U}_{2}+\mathrm{V}_{3}=\mathrm{C}_{23}=>5+\mathrm{V}_{3}=5 \quad \mathrm{~V}_{3}=0 \\
& \mathrm{U}_{2}+\mathrm{V}_{2}=\mathrm{C}_{22}=>\mathrm{U}_{2}+1=6 \quad \mathrm{U}_{2}=5 \\
& \mathrm{U}_{3}+\mathrm{V}_{3}=\mathrm{C}_{33}=>\mathrm{U}_{3}+0=3 \quad \mathrm{U}_{3}=3 \\
& \mathrm{U}_{3}+\mathrm{V}_{4}=\mathrm{C}_{34}=>3+\mathrm{V}_{4}=2 \mathrm{~V}_{4}=-1 \\
& \mathrm{P}_{13}=\mathrm{U}_{1}+\mathrm{V}_{3}-7 \Rightarrow 0+0-7 \Rightarrow \quad \mathrm{C}_{13}=-7 \\
& \mathrm{P}_{14}=\mathrm{U}_{1}+\mathrm{V}_{4}-4 \Rightarrow 0+(-1)-4 \Rightarrow \mathrm{C}_{14}=-5 \\
& \mathrm{P}_{21}=\mathrm{U}_{2}+\mathrm{V}_{1}-2 \Rightarrow 5+2-2 \Rightarrow \mathrm{C}_{21}=6 \text { Max } \\
& \mathrm{P}_{24}=\mathrm{U}_{2}+\mathrm{V}_{4}-4 \Rightarrow 5+(-1)-9 \Rightarrow \mathrm{C}_{24}=-5 \\
& \mathrm{P}_{31}=\mathrm{U}_{3}+\mathrm{V}_{1}-8 \Rightarrow>3+3-8 \Rightarrow \mathrm{C}_{31}=-2 \\
& \mathrm{P}_{32}=\mathrm{U}_{3}+\mathrm{V}_{2}-3=>3+1-3=>\mathrm{C}_{32}=1
\end{aligned}
$$

\begin{tabular}{|c|c|c|c|c|c|}
\hline & $V_{1}=-3$ & $\mathrm{~V}_{2}=1$ & $\mathrm{~V}_{3}=0$ & $\mathrm{~V}_{4}=-1$ & $S$ \\
\hline $\mathrm{U}_{1}=0$ & 3 & 1 & 7 & 4 & 250 \\
\hline $\mathrm{U}_{2}=5$ & $\sqrt{200}$ & $\begin{array}{|rr|}6 & (-) \\
& 50 \\
\end{array}$ & $\sqrt[5]{100}$ & 9 & 350 \\
\hline $\mathrm{U}_{3}=3$ & 8 & 3 & $\begin{array}{l}3 \\
3 \\
250\end{array}$ & $\stackrel{2}{150}$ & 400 \\
\hline $\mathrm{D}$ & 200 & 300 & 350 & 150 & \\
\hline
\end{tabular}

allocated cells.

Repeat the step \# 2 and step \# 3 
Using $U_{i}+V_{j}=C_{i j}$ for all allocated cells.

$$
\begin{aligned}
& \mathrm{U}_{1}+\mathrm{V}_{1}=\mathrm{C}_{12}=>0+\mathrm{V}_{2}=1 \mathrm{\textrm {V } _ { 2 } = 3} \\
& \mathrm{U}_{2}+\mathrm{V}_{2}=\mathrm{C}_{22}=>\mathrm{U}_{2}+1=1 \mathrm{U}_{2}=5 \\
& \mathrm{U}_{2}+\mathrm{V}_{3}=\mathrm{C}_{23}=>5+\mathrm{V}_{3}=5 \mathrm{~V}_{3}=0 \\
& \mathrm{U}_{2}+\mathrm{V}_{1}=\mathrm{C}_{21}=>5+\mathrm{V}_{1}=2 \mathrm{~V}_{1=-3} \\
& \mathrm{U}_{3}+\mathrm{V}_{3}=\mathrm{C}_{33}=>\mathrm{U}_{3}+0=3 \mathrm{U}_{3}=3 \\
& \mathrm{U}_{3}+\mathrm{V}_{4}=\mathrm{C}_{34}=>3+\mathrm{V}_{4}=2 \mathrm{~V}_{4=-1}
\end{aligned}
$$

\begin{tabular}{|c|c|c|c|c|c|}
\hline & $V_{1}=-2$ & $V_{2}=1$ & $V_{3}=1$ & $V_{4}=0$ & $S$ \\
\hline $\mathrm{U}_{1}=0$ & 3 & 1 & 7 & 4 & 250 \\
\hline $\mathrm{U}_{2}=4$ & $\frac{2}{200}$ & 6 & 5 & 9 & 350 \\
\hline $\mathrm{U}_{3}=2$ & 8 & $\frac{3}{50}$ & 200 & 2 & 400 \\
\hline $\mathrm{D}$ & 200 & 300 & 350 & 150 & \\
\hline
\end{tabular}

Now agin Find $\mathrm{P}_{\mathrm{ij}}=\mathrm{U}_{\mathrm{i}}+\mathrm{V}_{\mathrm{j}}-\mathrm{C}_{\mathrm{ij}}$ for all non Allocated cells

$$
\begin{aligned}
& \mathrm{P}_{11}=\mathrm{U}_{1}+\mathrm{V}_{1}-3=>0+(-3)-3 \Rightarrow \mathrm{C}_{11}=-6 \\
& \mathrm{P}_{13}=\mathrm{U}_{1}+\mathrm{V}_{3}-7=>0+01-7 \Rightarrow \mathrm{C}_{13}=-7 \\
& \mathrm{P}_{14}=\mathrm{U}_{1}+\mathrm{V}_{4}-4=>0+(-1)-4 \Rightarrow>\mathrm{C}_{14}=-5 \\
& \mathrm{P}_{24}=\mathrm{U}_{2}+\mathrm{V}_{4}-9 \Rightarrow>5+(-1)-9 \Rightarrow \mathrm{C}_{24}=-5 \\
& \mathrm{P}_{31}=\mathrm{U}_{3}+\mathrm{V}_{1}-8 \Rightarrow>3+(-3)-8 \Rightarrow \mathrm{C}_{31}=-8 \\
& \mathrm{P}_{32}=\mathrm{U}_{3}+\mathrm{V}_{2}-3=>3+1-3=>\mathrm{C}_{32}=1 \text { Max }
\end{aligned}
$$

\begin{tabular}{|c|c|c|c|c|c|c|}
\hline \multicolumn{7}{|c|}{ (1) } \\
\hline \multirow{5}{*}{ (5) } & 3 & 1 & 7 & 4 & 250 & \multirow{5}{*}{ (2) } \\
\hline & 2 & 6 & 5 & 9 & 350 & \\
\hline & \multirow[t]{2}{*}{8} & \multirow[t]{2}{*}{3} & \multirow[t]{2}{*}{3} & 2 & 400 & \\
\hline & & & & 150 & 250 & \\
\hline & 200 & 300 & 350 & $\begin{array}{c}150 \\
0\end{array}$ & & \\
\hline & & & (6) & & & \\
\hline
\end{tabular}

\section{Repeat the step \# 2 and step \# 3}

Now agin Find $\mathrm{P}_{\mathrm{ij}}=\mathrm{U}_{\mathrm{i}}+\mathrm{V}_{\mathrm{j}}-\mathrm{C}_{\mathrm{ij}}$ for all non Allocated cells.

$\mathrm{P}_{11}=\mathrm{U}_{1}+\mathrm{V}_{1}-3=>0+(-2)-5 \Rightarrow \mathrm{P}_{11}=-5 \quad \mathrm{P}_{13}=\mathrm{U}_{1}+\mathrm{V}_{3}-7 \Rightarrow>0+1-7 \Rightarrow \mathrm{C}_{13}=-6$

$\mathrm{P}_{14}=\mathrm{U}_{1}+\mathrm{V}_{4}-4 \Rightarrow 0+0-4 \Rightarrow \mathrm{P}_{14}=-4 \quad \mathrm{P}_{14}=\mathrm{U}_{1}+\mathrm{V}_{4}-4 \Rightarrow>0+0-4 \Rightarrow \mathrm{C}_{14}=-4$

$\mathrm{P}_{24}=\mathrm{U}_{3}+\mathrm{V}_{1}-8 \Rightarrow>4+0-9 \Rightarrow \mathrm{P}_{31}=-5 \quad \mathrm{P}_{31}=\mathrm{U}_{3}+\mathrm{V}_{2}-3=>2+(-2)-8=>\mathrm{C}_{31}=-8$

So all $\mathrm{P}_{\mathrm{ij}}=\mathrm{U}_{\mathrm{i}}+\mathrm{V}_{\mathrm{j}}-\mathrm{C}_{\mathrm{ij}} \leq 0$ is true Stop here

\section{Propose New Algorithm:-}

\section{$\mathbf{1}^{\text {st }}$ Iteration}

\section{$2^{\text {st }}$ Iteration}

\begin{tabular}{|c|c|c|c|c|c|}
\hline \multicolumn{7}{|c|}{ (4) } \\
\hline \multirow{4}{*}{} & 3 & 1 & 7 & 250 & \\
\cline { 2 - 5 } & 2 & 6 & 5 & 350 & \\
& 200 & & & 150 & \\
\cline { 2 - 5 }$(5)$ & 8 & 3 & 3 & 250 & \multirow{2}{*}{$(4)$} \\
\cline { 2 - 5 } & 200 & 300 & 350 & & \\
\hline & 0 & & & & \\
\hline
\end{tabular}




\section{$3^{\text {rd }}$ Iteration}

\begin{tabular}{|c|c|c|c|c|}
\hline & \multicolumn{3}{|c|}{ (6) } & \\
\hline \multirow[b]{5}{*}{ (2) } & 1 & 7 & 250 & \multirow[b]{5}{*}{ (4) } \\
\hline & 250 & & 0 & \\
\hline & 6 & 5 & 150 & \\
\hline & 3 & 3 & 250 & \\
\hline & $\begin{array}{l}300 \\
50\end{array}$ & 350 & & \\
\hline & . & (0) & & \\
\hline
\end{tabular}

\section{$5^{\text {th }}$ Iteration}

\begin{tabular}{|c|c|c|c|}
\hline & \multicolumn{2}{|l|}{ (5) } & \\
\hline \multirow{7}{*}{ (2) } & 5 & 150 & \multirow{7}{*}{ (2) } \\
\hline & 150 & 0 & \\
\hline & 3 & 200 & \\
\hline & 200 & 0 & \\
\hline & 350 & & \\
\hline & 150 & & \\
\hline & 0 & & \\
\hline 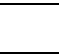 & (3) & & \\
\hline
\end{tabular}

\section{Result Analysis:}

\begin{tabular}{|l|c|c|c|c|}
\hline \multirow{2}{*}{ Transportation Problem } & \multicolumn{3}{|c|}{ Methods } \\
\cline { 2 - 5 } & MODI Optimal Solution & New method & LCM & NWCM \\
\hline Example-1 & 2450 & 2450 & 2450 & 3700 \\
\hline Example-2 & 2850 & 2850 & 2900 & 4400 \\
\hline
\end{tabular}

\section{Conclusion:-}

In this paper we introduce a new algorithm for optimal solution of Transportation Problem. Proposed research is very useful to get optimal solution directly without using M.O.D.I. method. M.O.D.I method is doubtful method for selecting which I.B.F.S to be applied, this method gets started from some other method like as (N.W.C.M),(L.C.M) \& (V.A.M). According to time point of view M.O.D.I method is more time consuming and more calculation required to get optimal solution. This Proposed method is easier than other methods to compute optimal solution and provides optimal solution without using I.B.F.S. We also check several examples other than above examples that most of time Propose algorithm provide optimal solution directly. 


\section{Reference:-}

[1] Hakim. M. A. (2012), “An Alternative Method to Find Initial Basic Feasible Solution of a Transportation Problem". Vol. 1, No. 2, 203-209.

[2] B.S Grewal "Higher Engineering Mathematics", (2012), 42 ${ }^{\text {nd }}$ Edition Page No: 1097 to1102, ISBN No: 978-81-7409-195-5.

[3] Md. Ashraful babu and Utpal Kanti Das, (2016), "Lowest Allocation Method (LAM)", International Journal of Scientific \& Engineering Research, Volume 4, Issue 11, ISSN 2229-5518.

[4] M.A. Metlo , M.A. Solangi and S.A. Memon, (2016) "Modified North West Corner" (M.N.W.C.M), Sindh Univ. Res. Jour. (Sci. Ser.) Vol. 48 (4) 793-796.

[5] Tuncay Can and Habip Koçak, (2016) “ Tuncay can's approximation method” Applied and Computational Mathematics. Vol. 5, No. 2, ISSN: 2328-5605.

[6] G.Kalpana and D.I.Geroge Amalarathinam, (2014), "Cost Effective Resource Allocation Method using Min---Min Algorithm in Grid Computing”, International Journal of Computer Applications (0975 - 8887) Volume 106 - No.7.

[7] Md. Ashraful Babu, Md. Abu Helal, Mohammad Sazzad Hasan \& Utpal Kanti Das, (2014), "Implied Cost Method (ICM)", Global Journals Inc. (USA), Volume 14 Issue 1 Version 1.0 , ISSN: 0975-5896.

[8] Abdul Sattar Soomro Muhammad Junaid \& Gurudeo Anand Tularam, (2015), "Modified Vogel's Approximation Method" (MVAM), Mathematical Theory and Modeling ISSN 2224-5804 (Paper) Vol.5, No.4.

[9] D. Almaatani, S.G. Diagne, Y. Gningue and P. M. Takouda, (2015), "Modified Vogel's Method" (MVM), Springer International Publishing Switzerland, DOI: 10.1007/978-3319-12307-3_3.

[10] Agarana M. C., Owoloko E. A. and Kolawole A. A., (2016) "Enhancing the Movement of People and Goods in A Potential World Class University using Transportation Model”, Global Journal of Pure and Applied Mathematics, ISSN 09731768 Volume 12, Number 1

[11] Md Sharif Uddin, Aminur Rahman Khan,Chowdhury Golam Kibria and Iliyana Raeva, (2016), "Improved Least Cost Method", Journal of Applied Mathematics \& Bioinformatics, vol.6, no.2, 1-20 ISSN: 1792-6602.

[12] Murthy, P. Rama. (2008) Operation Research, Second Edition, ISBN (13): 978-81224- 2944-2. 